\title{
The Impact Of Wavelet Selection On Transient Motor Current Signature Analysis
}

\author{
H Douglas $^{+}$, P.Pillay ${ }^{++}$ \\ ${ }^{+}$Clarkson University, on leave from the Department of Electrical Engineering \\ University of Cape Town, Rondebosch 7701, South Africa \\ Email:hdougla@eng.uct.ac.za \\ ${ }^{++}$Department of the Electrical \& Computer Engineering \\ Clarkson University, Potsdam, NY13699 \\ Email:pillayp@clarkson.edu
}

\begin{abstract}
This paper focuses on comparing the results using different wavelets for the detection of broken rotor bars in induction machines, operating under transient conditions. In Fourier analysis the basis functions are complex exponentials producing the same results for a particular waveform being analyzed. However in wavelet analysis the basis functions could be any permissible wavelets and the results produced are unique to the selected wavelet. Some wavelets are more efficient at encoding, denoising, compressing, decomposing and reconstructing signals than others. It is therefore desirable to select a wavelet that produces the best results for the signal being analyzed. The results show that using higher order wavelets improve the ability to detect broken rotor bars in induction machines operating under transient conditions.
\end{abstract}

\section{INTRODUCTION}

The detection of different machine faults, under steady state operating conditions, has improved over the past decade. Currently air gap eccentricities, bearing failures, inter-turn winding failures, as well as broken rotor bars can be detected by analyzing the frequency spectrum of the stator current of an induction machine [1-12].

It has been found that these steady state techniques are effective only when the machines, that are being diagnosed, are almost fully loaded and running at a constant speed. Subjecting a machine to heavy loads could be impractical and could reduce the operating lifetime of the machine. There are also cases where machines are never fully loaded. Because of these scenarios, the steady state techniques that have been previously developed, are less accurate when applied to machines that are lightly loaded or operate predominantly under transient conditions such as wind generators and motor operated valves etc.

An alternative to using steady state analysis has been proposed and researched in an attempt to detect machine faults by examining the starting transient of an induction machine. This approach is advantages because during startup the machine is subjected to stresses above normal operation. These stresses could highlight machine defects that are early in their development and not easily detected during steady state operation. The second advantage is that the machine is subjected to a wider range of slip, which further separates the frequencies associated with rotor bars. In this case the machine does not have to be heavily loaded in order to make an accurate diagnosis.

In [13] a method for the detection of broken rotor bars and end ring faults, by examining the starting transient is proposed. The detection algorithm is based on steady-state detection techniques by monitoring the sidebands of the current fundamental. Instead of using the Fourier transform to monitor the sideband frequencies, a Phase Vocoder [14] based on the Short-Time Fourier Transform is used to produce a time-frequency representation of the starting current.

The problem here is that the system is based on the ShortTime Fourier Transform. The transform divides a nonstationary signal into small windows of equal time. The Fourier Transform is then applied to the time segment being examined. As the width or support of the window function decreases, a smaller portion of the input signal is considered, guaranteeing a greater time localization of frequency components in the signal. As the support of the window function increases, more accurate information about frequencies within the window is increased, but the ability to determine the time when those transients occur within the input signal is lost. The reason that time information is lost is due to the Heisenberg Uncertainty Principle. It states that one cannot know the exact time and frequency components of a signal [15], [16].

Wavelet analysis was introduced to overcome the shortcomings of Fourier analysis. In Fourier analysis the basis functions are complex exponentials producing the same results for a particular waveform being analyzed. However in wavelet analysis the basis function could be any permissible wavelet and the results produced are unique to the selected wavelet. Some wavelets are more efficient at encoding, denoising, compressing, decomposing and reconstructing signals than others. It is therefore desirable to select a wavelet that produces the best results for the signal being analyzed.

In the following sections we apply the discrete wavelet transform to the starting current of an induction machine. It will be shown that higher order wavelets produce results that are easier to interpret. These results are shown as clusters of coefficients in the decomposition of the current signal. 


\section{WAVELET THEORY}

The orthogonal basis functions used in Wavelet analysis are families of scaling functions, $\phi(t)$, and associated wavelets, $\psi(t)$. The scaling function, $\phi(t)$, can be represented by the following mathematical expression:

$$
\phi_{j, k}(t)=\sum_{k} H_{k} \phi\left(2^{j} t-k\right)
$$

where,

$H_{k}$ represents the coefficients of the scaling function, $k$ represents a translation,

$j$ represents the scale.

Similarly, the associated wavelet $\psi(t)$, can be generated using the same coefficients as the scaling function.

$$
\psi_{j, k}(t)=\sum_{k}(-1)^{k} \sqrt{2} h_{1-k} \phi\left(2^{j} t-k\right)
$$

The scaling functions are orthogonal to each other as well as with the wavelet functions as shown in (3), (4). This fact is crucial and forms part of the framework for a multiresolution analysis.

$$
\begin{aligned}
\int_{-\infty}^{\infty} \phi(2 t-k) \cdot \phi(2 t-l) d t & =0 \quad \text { for all } k \neq l . \\
\int_{-\infty}^{\infty} \psi(t) \cdot \phi(t) d t & =0
\end{aligned}
$$

Using an iterative method, the scaling function and associated wavelet can be computed if the coefficients are known. Figure 1 shows the Daubechies 2 scaling function and wavelet.

A signal can be decomposed into approximate coefficients, $a_{j, k}$, through the inner product of the original signal at scale $j$ and the scaling function.

$$
\begin{gathered}
a_{j, k}=\int_{-\infty}^{\infty} f_{j}(t) \cdot \phi_{j, k}(t) d t \\
\phi_{j, k}(t)=2^{-j / 2} \phi\left(2^{-j} t-k\right)
\end{gathered}
$$
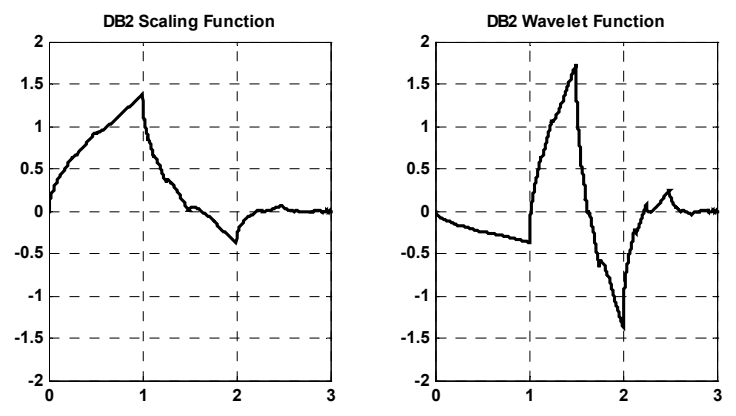

Figure 1. Daubechies2 scaling function (left) and associated wavelet (right).
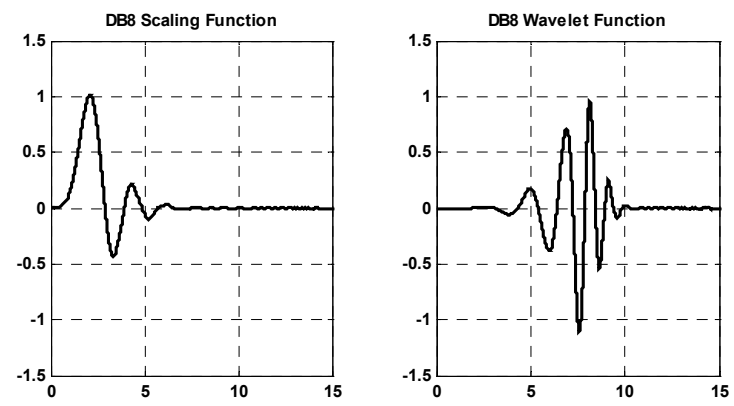

Figure 2. Daubechies8 scaling function (left) and associated wavelet (right).

Similarly the detail coefficients, $d_{j, k}$ can be obtained though the inner product of the signal and the complex conjugate of the wavelet function.

$$
\begin{aligned}
& d_{j, k}=\int_{-\infty}^{\infty} f_{j}(t) \cdot \psi_{j, k}^{*}(t) d t \\
& \psi_{j, k}(t)=2^{-j / 2} \psi\left(2^{-j} t-k\right)
\end{aligned}
$$

The original signal can therefore be reconstructed by a single series of scaling coefficients and a double series of the detail coefficients.

$$
f(t)=\sum_{j=-\infty}^{\infty} a_{j_{0}, k} . \phi_{j_{0}, k}+\sum_{j=-\infty}^{j_{0}} \sum_{k=-\infty}^{\infty} d_{j, k} . \psi_{j, k}(t)
$$

A discretized signal can be decomposed at different scales as follows:

$$
\begin{gathered}
f[n]=\sum_{k=0}^{N-1} a_{j, k} \phi_{j, k}(t) \\
=\sum_{k=0}^{N-1} a_{(j+1), k} \phi_{(j+1), k}(t)+\sum_{k=0}^{N-1} d_{(j+1), k} \psi_{(j+1), k}(t)
\end{gathered}
$$

The coefficients of the next decomposition level, $(j+1)$, can be expressed as

$$
\begin{gathered}
a_{(j+1), k}=\sum_{k=0}^{N} a_{j, k} \int \phi_{j, k}(t) \cdot \phi_{(j+1), k}(t) d t \\
d_{(j+1), k}=\sum_{k=0}^{N} a_{j, k} \int \phi_{j, k}(t) \cdot \psi_{(j+1), k}(t) d t \\
a_{(j+1), k}=\sum_{k} a_{j, k} \cdot g[k] \text { and } d_{(j+1), k}=\sum_{k} a_{j, k} \cdot h[k]
\end{gathered}
$$

The decomposition coefficients can therefore be determined through convolution and implemented by using a filter. The filter, $\mathrm{g}[\mathrm{k}]$, is a lowpass filter and $\mathrm{h}[\mathrm{k}]$ is a highpass filter.

$$
y[n]=\sum_{k=1}^{N} h[k] \cdot x[n-k]
$$



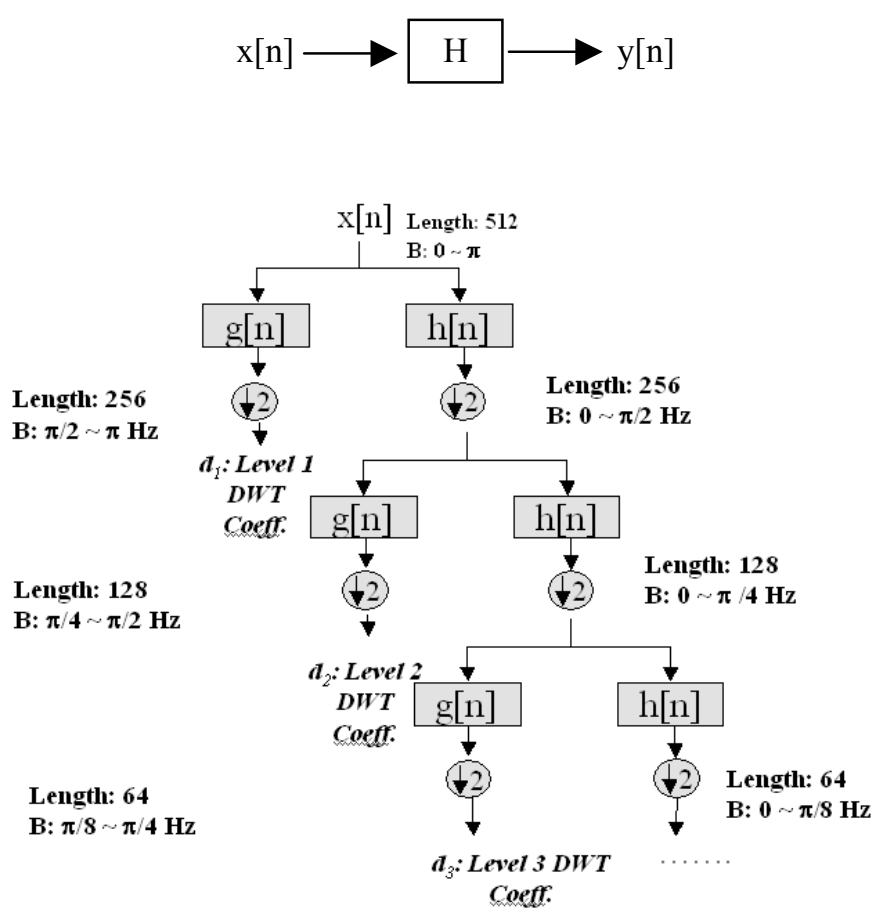

Figure 3. A three level multiresolution decomposition.

\section{SIGNAL PREPARATION FOR WAVELET ANALYSIS}

The nonstationary fundamental of the starting current needs to be removed before the wavelet analysis can be implemented. The individual measured line currents are transformed into a single rotating current vector as shown in figure 5 . The reason for combining the three line currents is that no two starting transients are exactly the same. The individual line currents will differ depending on instantaneous values of the line voltages at startup. When comparing the three line currents under different machine health conditions, it will be easier to compare a single rotating vector than three line currents that are not similar.

The vector is then transformed into the time domain and used as the input to an adaptive filter [18-19]. The starting current comprises two components i.e. the non-stationary fundamental and the residual after removing the fundamental from the total current.

The reason for using an adaptive filter can be justified as follows. We assume that the voltage waveform does not change in frequency or phase during the transient. If the power factor is measured, we'll find that it increases as the machine runs up. Since the power factor increases it can be concluded that the current has changed in frequency.

The residual waveform, after filtering, shown in figure 7 has information relating to the health of the machine including bad bearings, broken rotor bars etc.
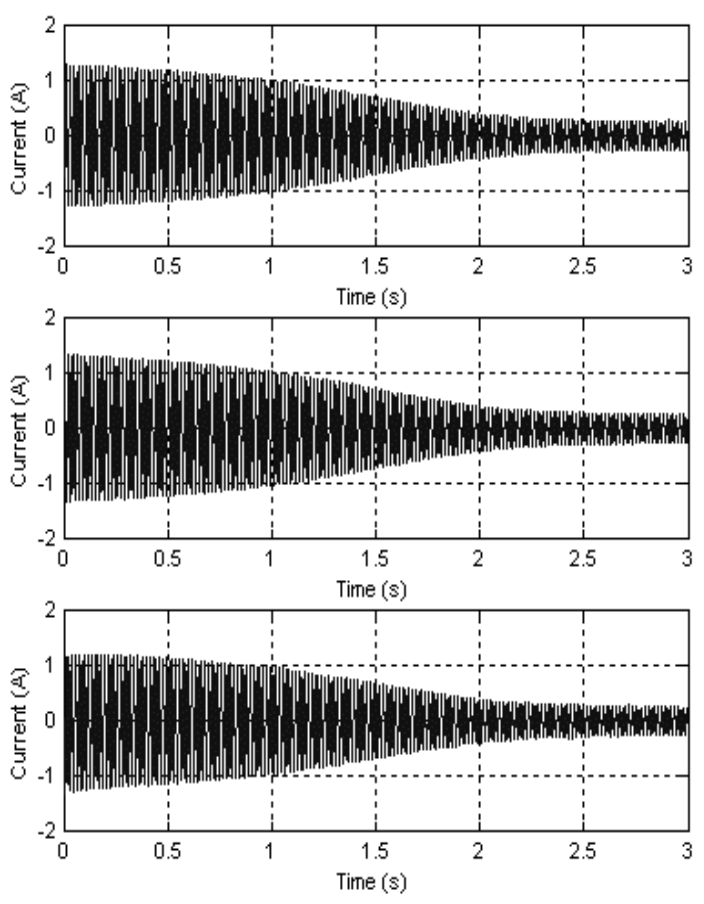

Figure 4. Startup current transients for phases A, B and C

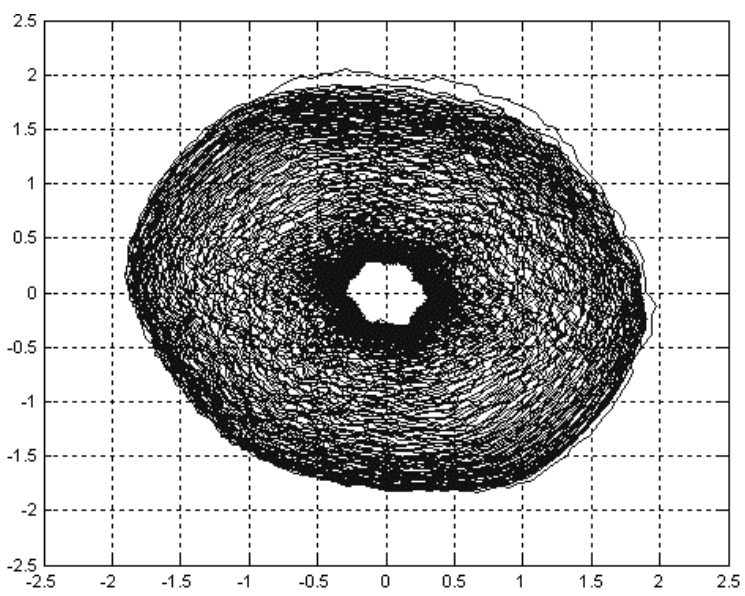

Figure 5. A plot of the current vector

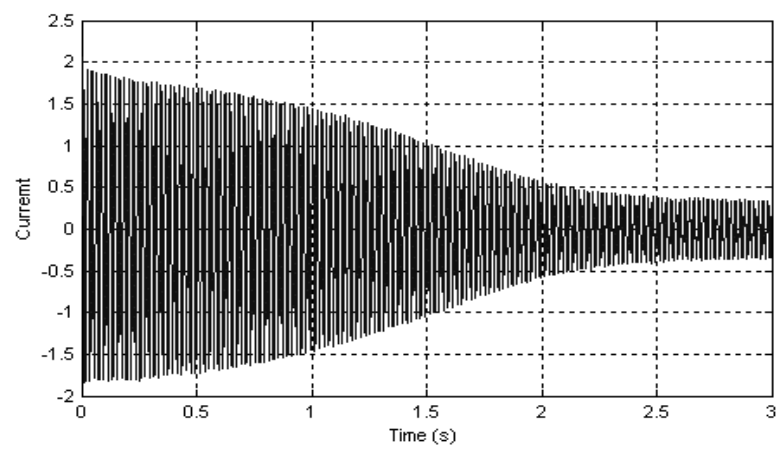

Figure 6. The time domain representation of the current vector. 


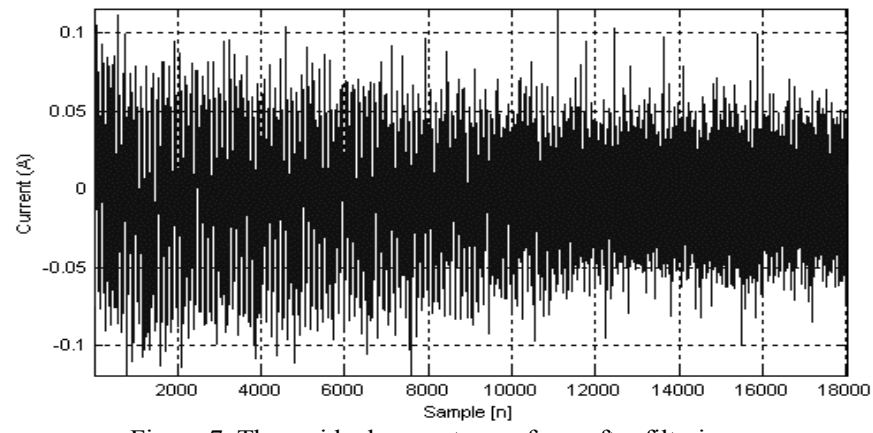

Figure 7. The residual current waveform after filtering.

\section{THE IMPACT OF DIFFERENT WAVELETS ON THE RESIDUAL CURRENT}

The methodology employed was to apply the discrete wavelet transform to the residual current. The family of Daubechies wavelets was chosen as the basis functions for the decomposition. Daubechies wavelets are classified according to the number of vanishing moments, $N$. The smoothness of the wavelets increases with the number of vanishing moments. For the case when $N=1$, the Daubechies scaling function and wavelet function resembles that of the Haar and are discontinuous. It is desirable to have smooth wavelets and therefore $N$ is increased. Although the Daubechies2 wavelet is continuous, its derivative is discontinuous. For $N$ greater than 2, the wavelet and its derivative are both continuous. It has been shown in applications such as compression, noise removal and singularity detection, that the number of vanishing moments plays a key role for efficient coding of signals [16].

In [20] it has been shown that the detail scale of interest for the detection of broken rotor bars is d9. Figures 9-10 show the ninth scale detail coefficients derived from wavelets Daubechies1 to Daubechies10 for a healthy machine and a machine with one broken rotor bar.

The results show that there is very little distinction between a healthy and damaged condition when comparing the scale $\mathrm{d} 9$ coefficients generated by DB1-DB3. For this reason these wavelets cannot be used in the detection analysis.

A distinct trend is observed when comparing the coefficients generated by DB4-DB7. In [20], it is shown that two groups of coefficients are dominant in the $9^{\text {th }}$ detail level.

The group of coefficients on the right hand side is only found in the presence of broken rotor bars as shown in figure 10. It should be noted that only in the coefficients generated by DB8-DB10, are two features present that are indicative of broken rotor bars. Firstly the group of coefficients on the right hand side is only present for the broken rotor bar data. Secondly, and more importantly, is the fact that there exists coefficients in the right-hand side group that are much larger than the coefficients in the left hand group. This is
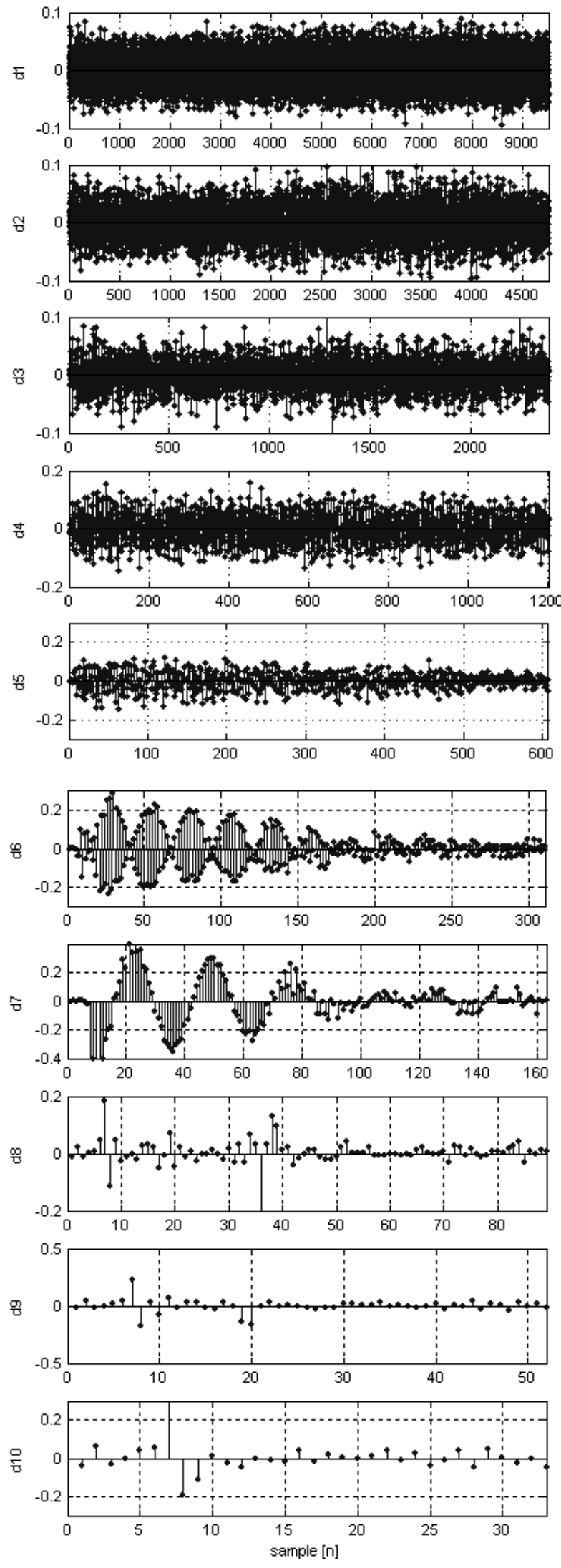

Figure 8. A 10 level decomposition of a healthy machine. 

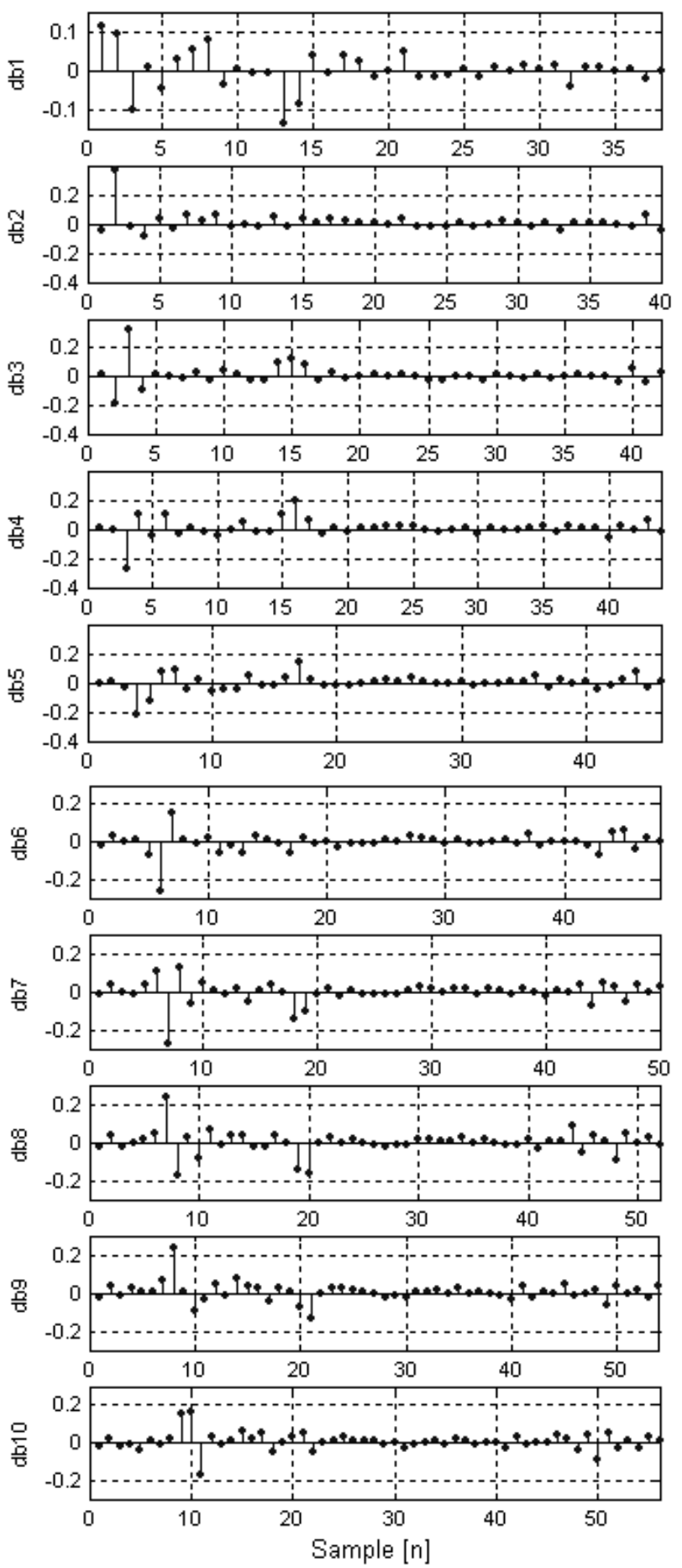

Figure 9. Wavelet decomposition of a healthy machine showing d9 using wavelets DB1-DB10.

particularly useful when dealing with ambiguous results such as the case of $60 \%$ loading where there seems to be a broken rotor bar present in the healthy data [20].

We can therefore conclude that the wavelet being used for the analysis should be at least of the order 8 i.e. DB8 in order to make an unambiguous diagnosis.
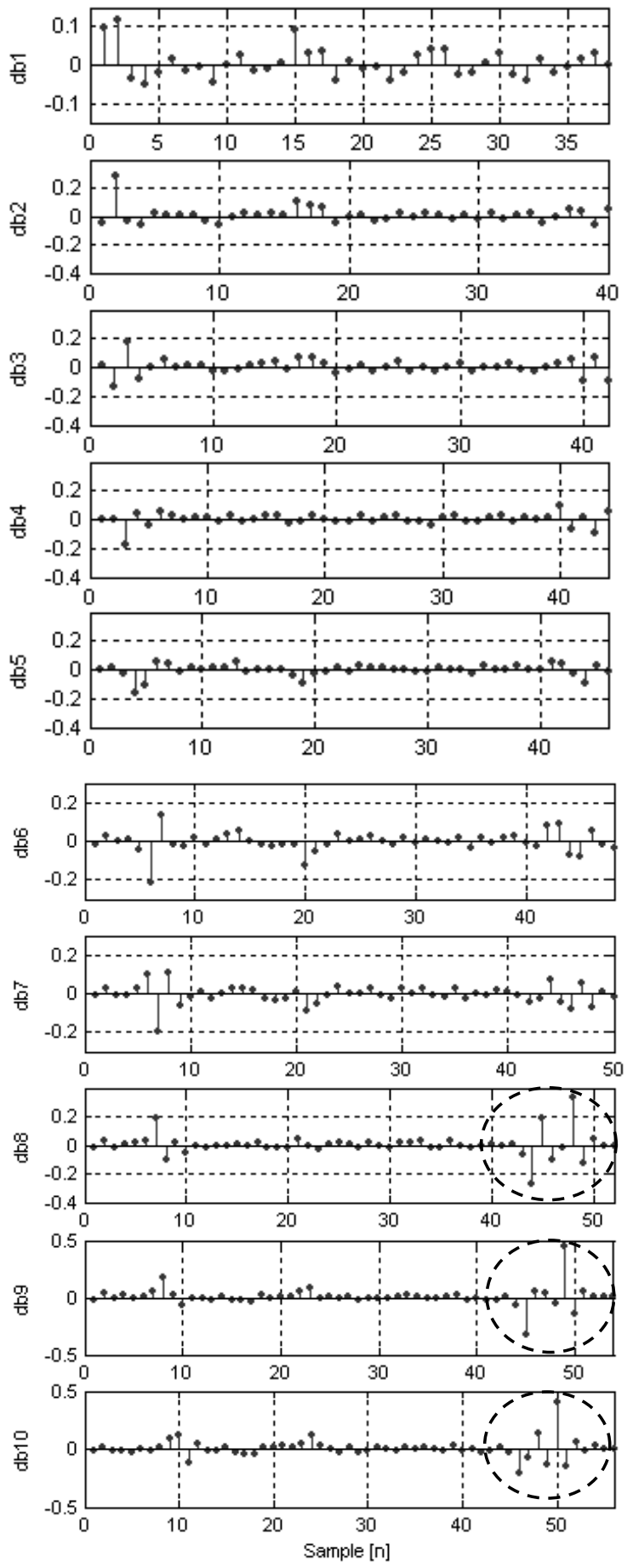

Figure 10.Wavelet decomposition of machine with one broken rotor bar showing d9 using wavelets DB1-DB10.

\section{CONCLUSIONS}

A wavelet analysis of the transient starting current of an induction machine can be used to detect the presence of broken rotor bars. From the results presented it is obvious that the choice of wavelet is crucial to the development of a fault detection algorithm. The number of vanishing moments 
affects the ability to distinguish between the conditions of the machine. The wavelets recommended for this application should be at least of order 8 to make an unambiguous assessment of the machines condition.

\section{ACKNOWLEDGMENTS}

The authors acknowledge the University of Cape Town Power Engineering Group for assistance with this project and the US Navy ONR for financial assistance.

\section{REFERENCES}

[1] G. B. Kliman et al., "Methods of motor current signature analysis,"Elect. Mach. Power Syst., vol. 20, no. 5, pp. 463-474, Sept. 1992.

[2] W. Deleroi, "Broken bars in squirrel cage rotor of an induction motorPart1: Description by superimposed fault currents", Arch. Elektrotech., vol. 67, pp. 91-99, 1984.

[3] R. Hirvonen, "On-line condition monitoring of defects in squirrel cage motors," in Proc. Int. Conf. Electrical Machines, vol. 2, 1994,Paris.

[4] Benbouzid \& M. Vieira, "Induction Motor Fault Detection and Localization Using Stator Current, Advanced Signal Processing Technique". IEEE Trans. Industrial Application, vol. 3, no. 1,1995

[5] S. L. Ho, W. L. Chan and H. W. Leung, "Application of Statistical Signal Processing for Condition Monitoring of Rotor Faults in Induction Motor" Electrical Machines and Drives, Sixth International Conference, pp. $97-102,1993$.

[6] G. B. Kliman, J. Stein, "Induction Motor Fault Detection Via Passive Current Monitoring a Brief Survey." pp. 49-65

[7] W. T. Thomson, I. D. Stewart, "On-line Current Monitoring for Fault Diagnosis in Inverter Fed Induction Motor", Life Management of Power Plants, 1994, International Conference, pp. 66- 73.

[8] C. Hargis et al., "The detection of rotor defects in induction motors," in Proc. 1982 IEE Int. Conf. Electrical Machines, Design and Application, London, U.K., pp. 216-220.
[9] M. E H. Benbouzid, "A Review of Induction Motors Signature Analysis as a Medium for Faults Detection", IEEE Transactions on Industrial Electronics, vol. 47, no. 5, October 2000

[10] W. Deleroi, "Squirrel Cage Motor with Broken Bar in the RotorPhysical Phenomena and their Experimental Assessment", Proc. ICEM'82. Budapest, Hungary, 1982, pp. 767-770.

[11] H. A. Toliyat et al., "Condition monitoring and fault diagnosis of electrical machines-A review," in Conf. Rec. 1999 IEEE-IAS Annual. Meeting, vol. 1, Phoenix, pp. 197-204.

[12] F. Filippetti et al., "AI techniques in induction machines diagnosis including the speed ripple effect," IEEE Trans. Ind. Applications, vol. 34, pp. 98-108, Jan./Feb. 1998.

[13] J. F. Watson and S Elder, "Transient analysis of line current as a fault detection technique for 3-phase induction machines", International conference of Electrical Machines, 1992

[14] Schafer R. W., Rabiner L.R. "Design and analysis of a speech analysissynthesis system based on short time Fourier analysis"

[15] O. Rioul and M. Vettrli, "Wavelet and Signal Processing", IEEE SP Magazine, October 1991, pp.14-38

[16] S. G. Mallat, "A Wavelet Tour of Signal Processing", Academic Press, 1998.

[17] M. Haji, H. A. Toliyat, "Pattern Recognition - A Technique for Induction Machines Rotor Fault Detections," Proceedings of the IEEE IEMDC' 01, Boston, MA, June 17-20, 2001.

[18] Ziarani, A.K.; Konrad, A.” A nonlinear adaptive method of elimination of power line interference in ECG signals" "IEEE Transactions on Biomedical Engineering, vol 49, Issue: 6, June 2002

[19] M. Karimi-Ghartemani and A. K. Ziarani, "Periodic orbit analysis of two dynamical systems for electrical engineering applications," Journal of Engineering Mathematics, vol. 45, no. 2,2003, pp. 135-154.

[20] H. Douglas, P. Pillay and A K Ziarani, "A new algorithm for transient motor condition monitoring", IEEE Transactions on Industry Applications, vol. 40, no. 5, Sept-Oct, 2004.

[21] P.Pillay and Z.Xu, "Motor Current Signature Analysis", IEEE IAS Annual Meeting, Oct 1996 